\title{
STUDY OF SECONDARY PHASES IN TRIP STEEL BY ADVANCED SEM AND AFM TECHNIQUES
}

\author{
'Šárka MIKMEKOVÁ, ²Ondřej AMBROŽ, ${ }^{3}$ Veronika HEGROVÁ, ${ }^{4}$ Tomohiro AOYAMA \\ 'ISI Brno, Czech Academy of Sciences, Brno, Czech Republic, EU, sarka@isibrno.cz \\ 2ISI Brno, Czech Academy of Sciences, Brno, Czech Republic, EU, ondrej@isibrno.cz \\ ${ }^{3}$ NenoVision s.r.o., Brno, Czech Republic, EU, veronika.hegerova@nenovision.com \\ 4JFE Steel Research Laboratory, JFE Steel Corporation, Fukuyama, Japan, to-aoyama@ife-steel.co.jp
}

https://doi.org/10.37904/metal.2020.3516

\begin{abstract}
The paper aims to demonstrate a modern scanning electron microscope (SEM) as a powerful tool for visualization of the secondary phases in TRIP steel. The TRIP steel specimens prepared by various metallographic techniques were imaged by the SEM and the secondary phases presence was confirmed by an electron back-scattered diffraction (EBSD) technique. The chemical polishing by $5 \% \mathrm{HF}$ in $\mathrm{H}_{2} \mathrm{O}_{2}$ for 10 seconds results in selective etching for each individual phase, as confirmed by an atomic force microscopy (AFM) and hybrid AFM-in-SEM techniques. The phases are easily distinguishable in the SEM micrographs created by the low energy high take-off angle signal electrons. The proposed sample preparation technique together with special SEM imaging conditions enables us accurate analysis of distribution of secondary phases within the TRIP steel matrix and moreover, the retained austenite is distinguishable from the martensite phase.
\end{abstract}

Keywords: TRIP steel, advanced SEM, metallography

\section{INTRODUCTION}

The understanding of the relationship between phase composition and macroscopic properties plays a key role in the development of advanced high strength steels, such as transformation induced plasticity (TRIP) steels. Advanced TRIP steels have complex microstructure consisting of ferrite-bainite matrix and secondary phases (martensite and retained austenite) inhomogeneously dispersed within a matrix [1]. Analysis of advanced TRIP steels phase composition is very complicated due to presence of very fine secondary phases, such as lath-like austenite. An electron back-scattered diffraction technique (EBSD) is a common tool enabling us phase analysis. The EBSD technique is able to distinguish the phases based on their crystallographic lattice (austenite) and image quality (martensite) [2,3]. On the other hand, the EBSD technique is not effective for characterization of large area and very fine phases. The scanning electron microscopy (SEM) seems be as a useful tool for phase characterization due to a possibility of large area mapping in short time and high spatial resolution. Unfortunately, standard metallographic techniques are not able to prepare the surface specific for each phase and the secondary phases are not distinguishable in ordinary SEM micrographs. The aim of this work is to look into the commonly used metallographic sample preparation techniques and explore their potentials to prepare the surface unique for each phase.

\section{EXPERIMENTAL}

The material under investigation was a TRIP steel containing $12 \%$ of retained austenite (measured by X-Ray diffraction technique). The TRIP steel consists of ferrite-bainite matrix and contains the secondary phases, 
such as retained austenite, martensite and marteniste-austenite constituents. The specimens were prepared conventionally by grinding and mechanical polishing. The first specimen was only mechanically polished and the final step of polishing was $0.25 \mu \mathrm{m}$ diamond paste for 10 minutes. The second specimen surface was finished by chemical-mechanical polishing using $40 \mathrm{~nm} \mathrm{SiO} 2$ particles. Polishing with colloidal silica lasted for 3 hours. The third specimen was chemically polished by means of immersion of the specimen into the $5 \% \mathrm{HF}$ in $\mathrm{H}_{2} \mathrm{O}_{2}$ for 10 seconds. The last specimen was prepared by conventional electro-polishing technique. The specimens were characterized by a dualbeam FIB-SEM Helios 4G HP (Thermofisher Scientific) [4]. This instrument is equipped with highly sophisticated detection system enabling effective energy and angular filtering of the signal electrons. The microscope is equipped with several detectors, such as in-chamber (ETD, ICE) and in-lens (TLD, MD) secondary electron detectors and a concentric back-scattered electron detector (CBS) consisting of four concentric parts, which can be operated individually. The instrument can be operated at very low landing energies of the primary beam due to a specimen bias mode. The negative specimen bias accelerates the low emission energy electrons towards the detectors and collimated their trajectories towards the optical axis. It secures us high detection efficiency at low landing energies [5,6]. The surface morphology was characterized by an atomic force microscope, LiteScope ${ }^{T M}$ (NenoVision), integrated into SEM. This approach enables precise AFM tip navigation by SEM and simultaneous AFM and SEM data acquisition and their precise correlation (LiteScope ${ }^{\mathrm{TM}}$, Nenovision) [7]. The EBSD analysis was performed by the EDAX Team software installed in the UHR SEM Magellan 400L (Thermofisher Scientific Company) [8].

\section{RESULTS AND DISCUSSION}

Figure 1 shows the EBSD phase maps and corresponding SEM secondary electrons (SE) micrographs of the TRIP steel specimens prepared by different ways. Obviously, the sample preparation finished with mechanical polishing results in austenite phase transformation and the secondary phases are not identifiable in the SEM image. The chemical-mechanical polishing with colloidal silica leads to perfectly smooth surface and the secondary phases are perfectly indexed in the EBSD phase map. On the other hand, absence of surface topography makes the phases undistinguished in the SEM image. The specimens prepared by chemical- and electro-polishing techniques enable to visualize the secondary phases in the SEM images. Moreover, the specimen prepared by chemical polishing exhibits the specific contrast for each secondary phase and the retained austenite becomes distinguishable from the martensite phase.

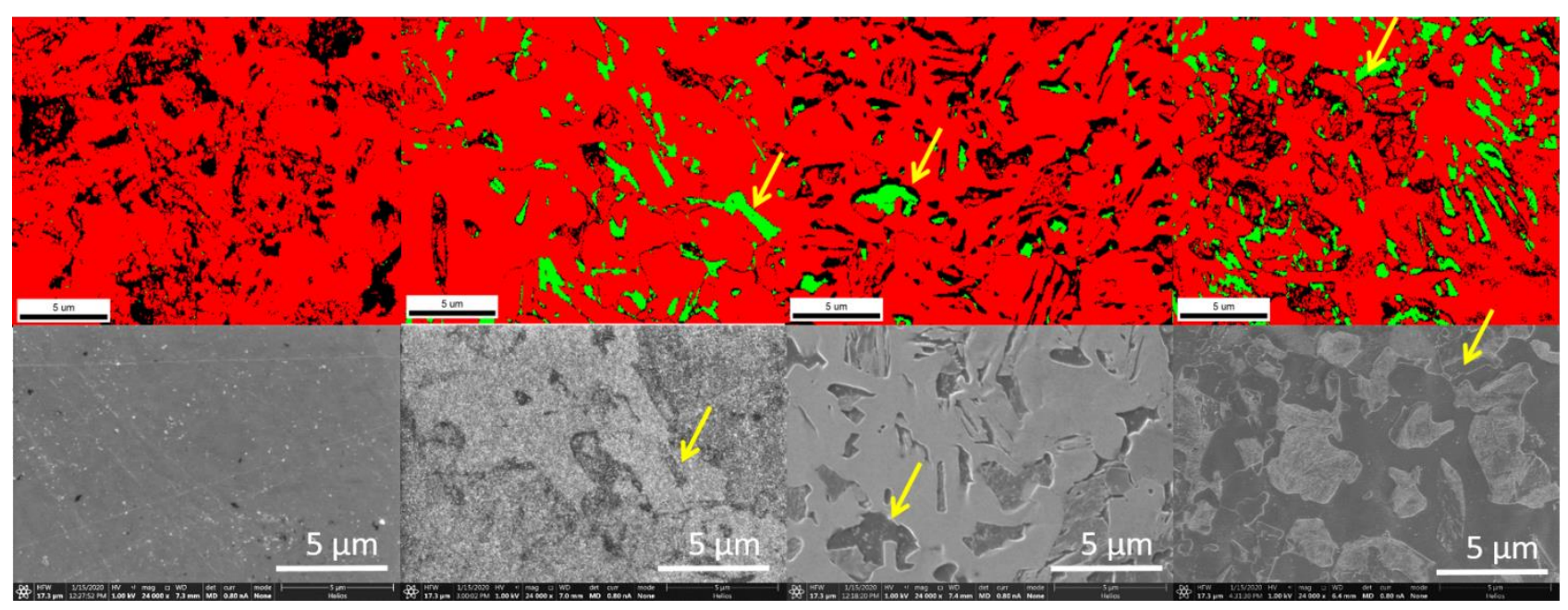

Figure 1 TRIP steel surface prepared by: simple mechanical polishing, chemical-mechanical polishing, chemical polishing and electro-polishing technique (from the left). Top row: EBSD phase map (red: BCC lattice - ferrite, martensite, green: FCC lattice - austenite); bottom row: SEM images taken by the in-lens SE detector. 
The specimen finished by chemical polishing appears to be promising for the secondary phase imaging by the SEM. Therefore, the next part of the SEM investigation will be focused on this specimen. Figure 2 shows a schematic sketch of the FIB-SEM Helios 4G detection system with utilized detectors of the signal electrons. The micrographs were collected at $1 \mathrm{keV}$ landing energy, which secures us to obtain high surface sensitivity. The primary electrons were retarded on the final landing energy by means of the electrostatic field created between the negatively biased specimen and the earthed detector. The signal electron trajectories are collimated towards the optical axis. The electrons are separated based on their emission energy and angle. The electrons having low emission energy and/or leave the specimen surface with the angle close to the optical axis are detected by the MD detector and the segment A of the CBS detector. Conversely, the elastically scattered electrons emitted under high angles from the optical axis are collected by the CBS segments situated far from the optical axis. As visible, filtering of the signal electrons helps us to amplify desired contrast. The images of MD and CBS-A were able to identify the three phases in the steel. The ferrite phase is the brightest, the austenite phase is the darkest, and the martensite phase is the second darkest. In the images of CBS-B, $C$, and $D$, the ferrite phase and the other phases can be distinguished, but the austenite phase and the martensite phase cannot be distinguished because they have the same brightness.

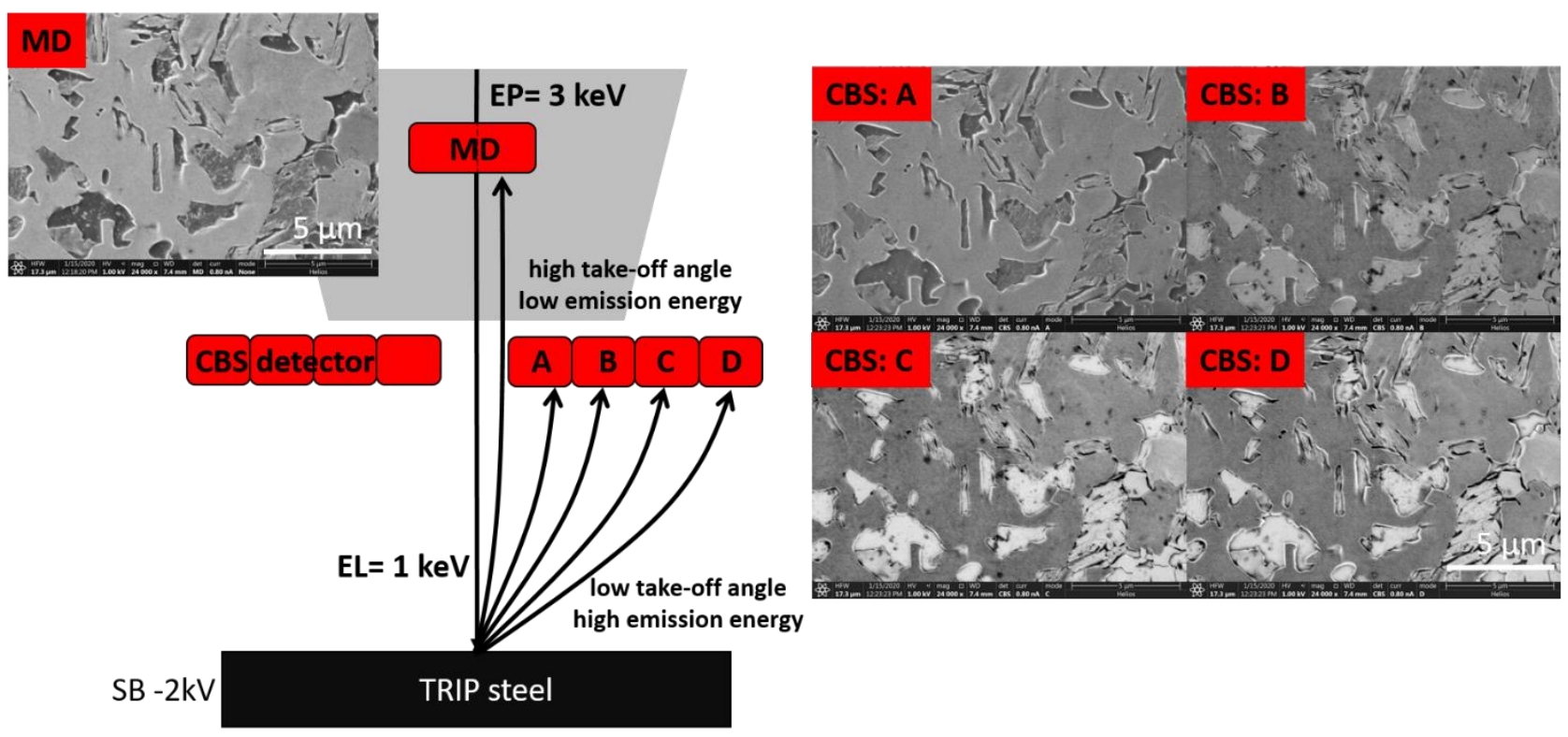

Figure 2 SEM micrographs obtained at $1 \mathrm{keV}$ landing energy of the primary electrons by the MD detector and by each individual segment of the CBS detector.

The SEM micrographs obtained at $1 \mathrm{keV}$ landing energy by the MD and the CBS D detectors are displayed in Figure 3, together with corresponding EBSD and AFM topography maps. Contrast in the SEM images corresponds with the topography of the surface confirmed by the AFM. The etching process is selective and the secondary phases are etched preferentially. The etching process is the most intensive in the case of the austenite phase. The austenite phase is dark in the SEM MD image. The SEM CBS D image shows the secondary phases brighter than the matrix and the austenite and the martensite phase are inseparably. Figure 4 compares the ordinary SEM SE obtained by conventional in-chamber ETD detector and the in-lens SEM MD detector. Both images are created mainly by the SE electrons. The ordinary SEM SE image is created by the low take-off angle electrons with contribution of the SE3 (the secondary electrons generated inside the chamber) and in contrast, the advanced SEM SE image obtained by the MD detector is created dominantly by the very low energy high take-off angle signal electrons. As visible, the advanced SEM SE image enables us to distinguish the secondary phases and the retained austenite becomes clearly visible. 

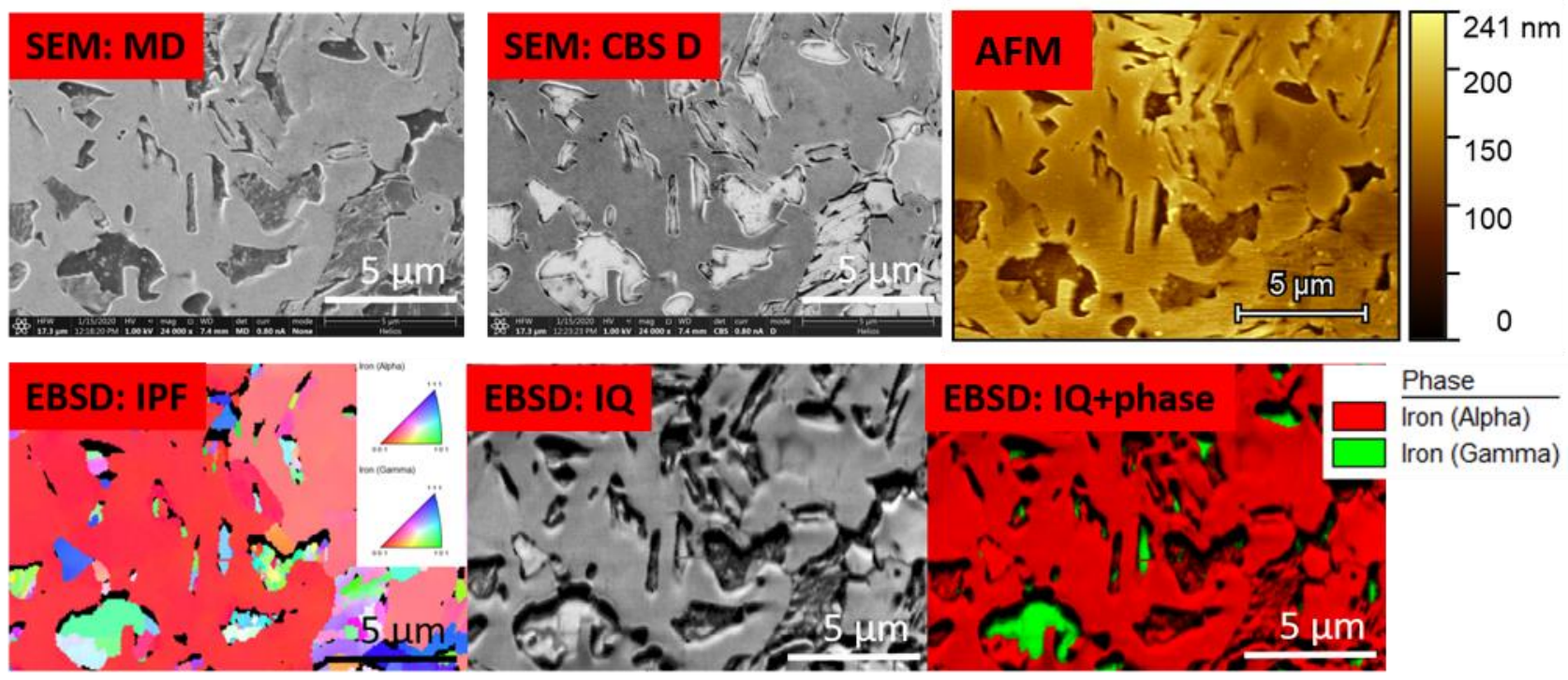

Figure 3 SEM micrographs created by the high take-off angle + low emission energy (SEM: MD) and by the low take-off angle high emission energy (SEM: CBS D) signal electrons, together with corresponding AFM topography (AFM) and EBSD results (crystal orientation map, image quality map and phase map).
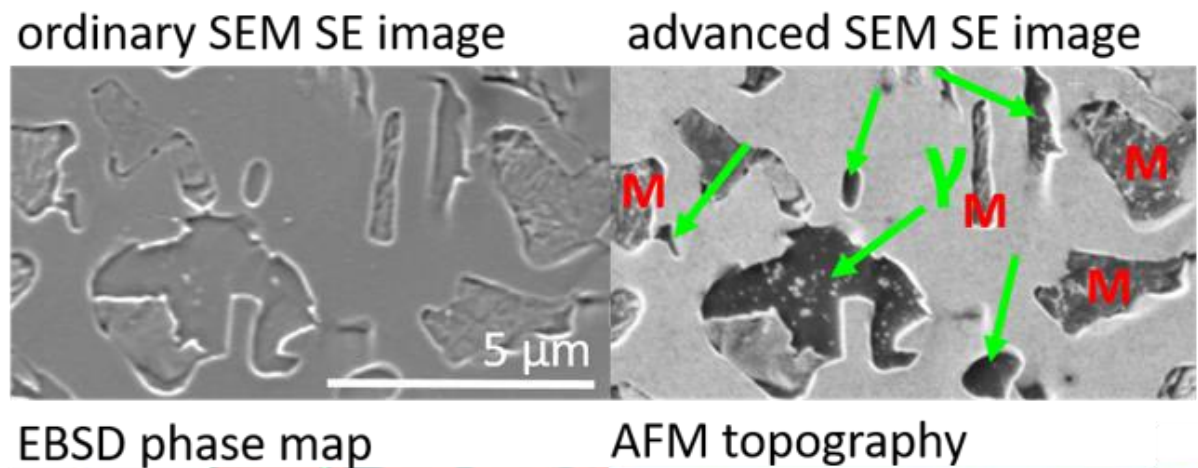

AFM topography

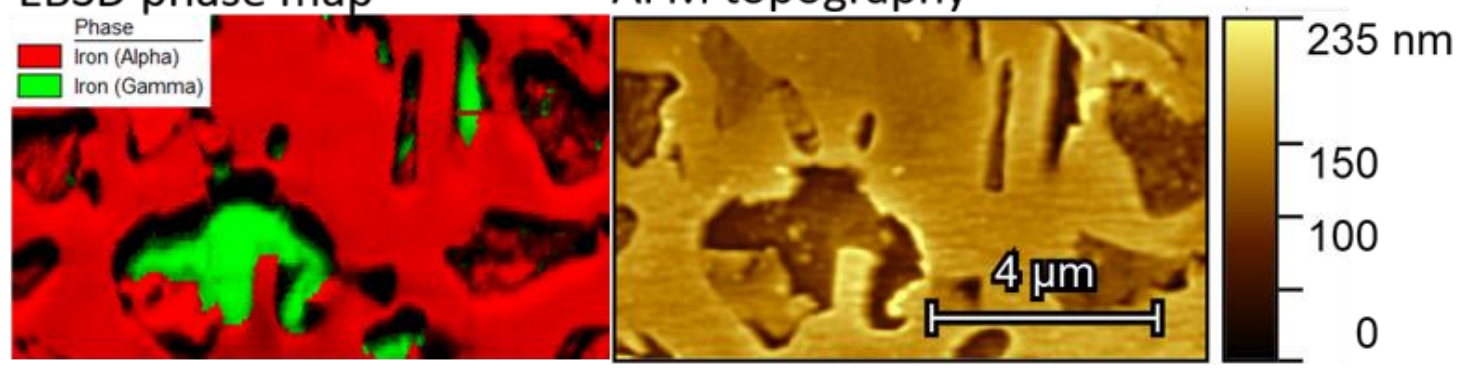

Figure 4 Contrast between the phases in the ordinary SEM SE image and the image obtained using effective angular and energy signal electrons filtering advanced SEM SE image, together with corresponding EBSD phase map and AFM topography.

Comprehensive information about TRIP steel surface morphology was obtained by means of special AFM LiteScopeTM, integrated directly into the SEM. This approach enabled precise AFM topographical measurement of the exact sample area previously analyzed by EBSD and advanced SEM for accurate data comparison. Also, LiteScopeTM is equipped with unique advanced correlative probe and electron microscopy (CPEM) imaging technology that enables simultaneous acquisition of AFM and SEM signals and their correlation into 3D CPEM view. As shown in Figure 5, material contrast (with focus on secondary phase) can be directly linked to the sample topography by this way. 


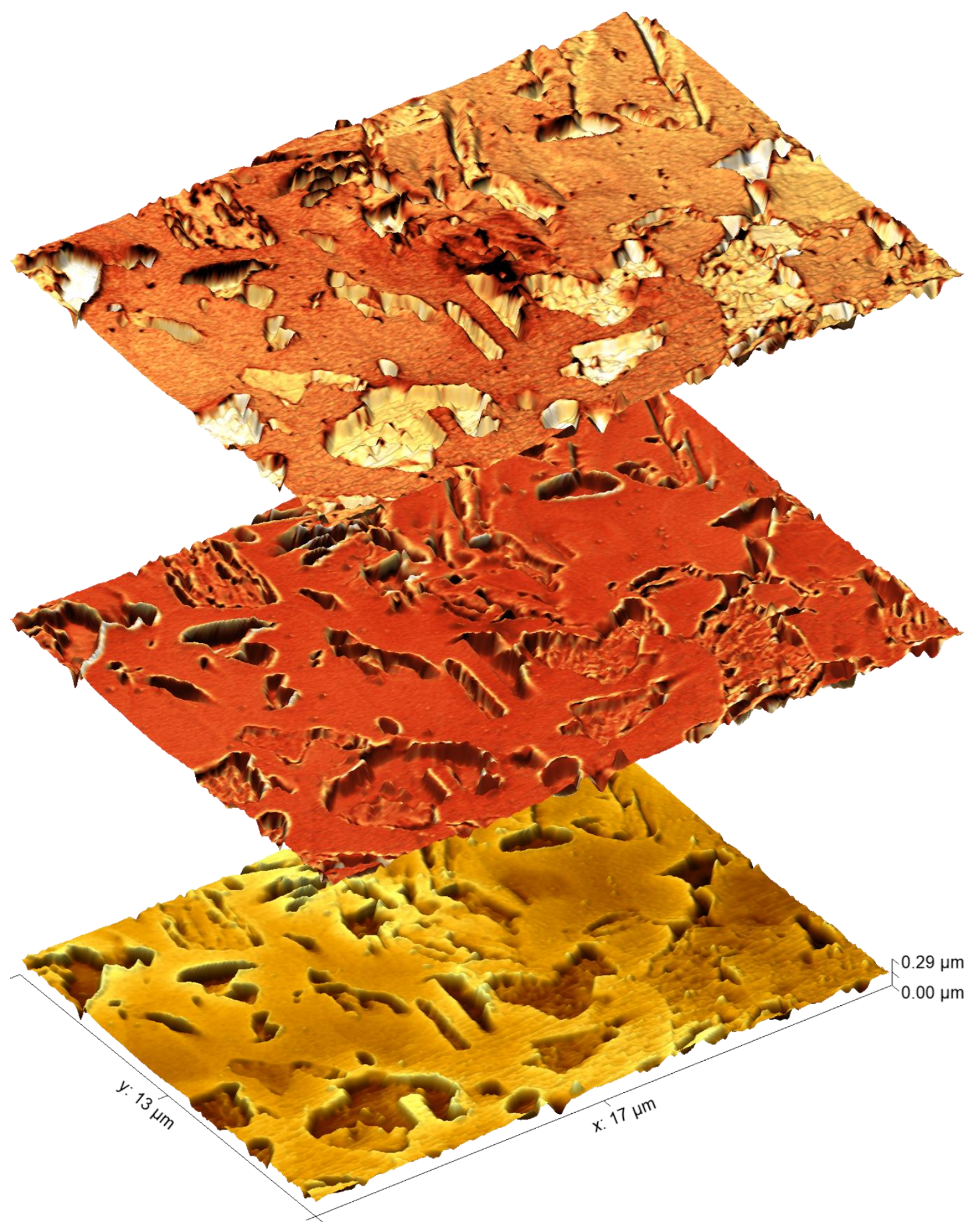

Figure 5 CPEM view of the TRIP steel specimen prepared by chemical etching technique: AFM + SEM BSE, AFM + SEM SE and AFM topography (from top to bottom).

\section{CONCLUSION}

Visualization of the secondary phases in TRIP steel by the SEM technique requires not only specially prepared specimen surface but also the possibility of effective energy and angular filtering of the signal electrons. Chemical polishing of the TRIP steel surface by $5 \% \mathrm{HF}$ in $\mathrm{H} 2 \mathrm{O} 2$ results in the selective etching process and 
the secondary phases are etched preferentially. The highest etch rate is in the case of austenite phase, as confirmed by the AFM topography imaging. The austenite phase shows specific contrast in the SEM micrographs created by the high take-off angle low energy signal electrons, which are collected by the in-lens SE detector. The surface sensitivity of the SEM technique is secured by using the low landing energies of the primary beam. Accurate information about the surface morphology was obtained by means of the correlative CPEM technique.

\section{ACKNOWLEDGEMENTS}

The research was supported by Technology Agency of the Czech Republic (TN01000008).

\section{REFERENCES}

[1] www.worldautosteel.org

[2] ZHANG, Y., LAI, P., JIA, H., XINHUA, J., CUI, G. Investigation of test parameters on EBSD analysis of retained austenite in TRIP and pipeline steels. Metals, 2019, vol. 9, no. 1, pp. 94-100.

[3] MAN, O., PANTELEJEV, L., PESINA, Z. EBSD analysis of phase composition of TRIP steel on various strain levels. Materials Engineering, 2009, vol. 16, no. 2, pp. 15-21.

[4] Helios NanoLab user operation manual (Thermofisher Scientific).

[5] MULLEROVÁ, I., FRANK, L. Scanning low energy electron microscopy. Advanced Imaging Electron Physics. 2003, vol. 128, pp. 308-443.

[6] MULLEROVÁ, I., LENC, M. Some approaches to low scanning electron microscopy. Ultramicroscopy, 1992, vol. 41, pp. 399-410.

[7] www.nenovision.com

[8] www.edax.com 